\title{
NMDA Receptors, mGluR5, and Endocannabinoids are Involved in a Cascade Leading to Hippocampal Long-Term Depression
}

\author{
Yukitoshi Izumi*,' and Charles F Zorumski ${ }^{1,2}$ \\ 'Departments of Psychiatry, Washington University School of Medicine, St Louis, MO, USA and ${ }^{2}$ Department of Neurobiology, Washington \\ University School of Medicine, St Louis, MO, USA
}

\begin{abstract}
In the CAI region of the rat hippocampus, metabotropic glutamate receptor-5 (mGluR5) and cannabinoid-I receptors (CBIRs) are believed to participate in long-term synaptic depression (LTD). How mGluRs and CBIRs interact to promote LTD remains uncertain. In this study, we examined LTD induced by CBIR agonists, mGluR5 agonists, and low-frequency electrical stimulation (LFS) of the Schaffer collateral pathway. Synthetic CBIR agonists induced robust LTD that was mimicked by the endocannabinoid (EC), noladin ether (NLDE), but not by anandamide. 2-Arachidonylglycerol (2AG) produced only a small degree of LTD. The selective mGluR5 agonist, namely (RS)-2-chloro-5-hydroxyphenylglycine (CHPG), also induced robust LTD, and CHPG and NLDE occluded each other's effects. Similarly, CHPG and NLDE occluded LFS-induced LTD, and LTD resulting from all three treatments was blocked by a CBIR antagonist. CHPG-LTD and NLDE-LTD were insensitive to N-methyl-D-aspartate receptor (NMDAR) block, even though LFS-LTD requires NMDARs. LTD induced by LFS or CHPG, but not NLDE-LTD, was blocked by a selective mGluR5 antagonist. (RS)-3,5dihydroxyphenylglycine (DHPG), a less selective group I mGluR agonist, also induced LTD, but its effects were not blocked by mGluR5 or CBIR antagonists. Furthermore, DHPG-LTD was additive with LFS-LTD and CHGP-LTD. These results suggest that NMDARs, mGluR5, and CBIRs participate in a cascade that underlies LFS-LTD and that release of an EC and CBIR activation occur downstream of NMDARs and mGluR5. Furthermore, DHPG induces a form of LTD that differs mechanistically from LFS-induced depression.

Neuropsychopharmacology (2012) 37, 609-617; doi:10.1038/npp.201 I.243; published online 12 October 20II
\end{abstract}

Keywords: synaptic plasticity; learning and memory; CBI receptors; metabotropic glutamate receptors; NMDA receptors; retrograde messengers

\section{INTRODUCTION}

Long-term depression (LTD) is a form of synaptic plasticity that likely contributes to learning and memory (Kemp and Manahan-Vaughan, 2004). In the early 1990s, several groups (Dudek and Bear, 1992; Fujii et al, 1991; Staubli and Lynch, 1990) reported that repeated low-frequency stimulation (LFS) of the Schaffer collateral pathway induced persistent homosynaptic depression of trans-synaptic responses. Early studies have demonstrated the importance of $N$-methyl-Daspartate receptors (NMDARs) in LTD induction (Dudek and Bear, 1992; Fujii et al, 1991), whereas subsequent work found that certain metabotropic glutamate receptors (mGluRs) also contribute, and can drive LTD by themselves under some conditions (Bellone et al, 2008; Fitzjohn et al, 1999). Thus, as is the case with long-term potentiation

\footnotetext{
*Correspondence: Dr Y Izumi, Department of Psychiatry, Washington University School of Medicine, 660 South Euclid Avenue, St Louis, MO 63110, USA, Tel: + I 314747 2987, Fax: + I 314 747 1896, E-mail: izumiy@wustl.edu

Received 15 April 2011; revised 16 August 2011; accepted 13 September 2011
}

(LTP) at Schaffer collateral synapses, both NMDARs and mGluRs are involved in the LTD induction process (Stanton et al, 1991; Bolshakov and Siegelbaum, 1994; Bashir et al, 1993; Wang et al, 2008, but see Selig et al, 1995; Overstreet et al, 1997). Among mGluRs, mGluR5 seems to be a key contributor (Reyes-Harde and Stanton, 1998) but, until recently, the availability of selective agonists and antagonists has limited studies of mGluR subtypes. LTD is now known to be expressed widely at excitatory synapses throughout the brain, although induction and expression mechanisms can vary depending on the region and stimulus used to induce LTD (Malenka and Bear, 2004; Massey and Bashir, 2007). At many excitatory synapses, LTD involves NMDAR activation, calcium influx, and early activation of protein phosphatases. Expression of LTP involves trafficking of postsynaptic AMPA-type glutamate receptors, although presynaptic changes are also observed (Malenka and Bear, 2004; Massey and Bashir, 2007; Poschel and Stanton, 2007).

In addition to synaptically driven, LFS-induced homosynaptic LTD, LTD can be induced pharmacologically by (RS)-3,5-dihydroxyphenylglycine (DHPG), an agonist of group I mGluRs that includes mGluR1 and mGluR5 
(Palmer et al, 1997; Fitzjohn et al, 1999; Huber et al, 2001; Gladding et al, 2009). However, DHPG-LTD can be induced even after NMDAR-dependent LTD has been established, and DHPG-LTD is not blocked by NMDAR antagonists. These findings suggest that DHPG-mediated LTD is distinct from conventional LFS-LTD. Whereas DHPG-LTD has been widely studied, (RS)-2-chloro-5-hydroxyphenylglycine (CHPG), a specific mGluR5 agonist (Doherty et al, 1997), has been used less often for LTD studies (Palmer et al, 1997; Lanté et al, 2006; Neyman and Manahan-Vaughan, 2008). Similarities and differences between CHPG- and DHPGinduced LTD have not been fully addressed, although CHPG-LTD cannot be induced after LFS-LTD (Lanté et al, 2006).

LFS-induced LTD can also involve the production of endocannabinoids (ECs). Notably, heterosynaptic LTD in the visual cortex is blocked by 2-methyl-6-(phenylethynyl)pyridine hydrochloride (MPEP), a relatively selective mGluR5 antagonist, and by AM251, an inhibitor of cannabinoid-1 receptors (CB1Rs), suggesting that release of ECs and activation of CB1Rs are involved in LTD induction after mGluR5 activation (Huang et al, 2008). Similarly, ECs are believed to participate in LTD induction in the striatum by serving as retrograde messengers (Gerdeman et al, 2002). Although the involvement of ECs in LTD has also been shown in the hippocampus during development (Yasuda et al, 2008) and in vivo (Abush and Akirav, 2010), a role for ECs in mGluR5-dependent homosynaptic LTD in the hippocampus has not been extensively investigated. It is also unclear which specific ECs participate in hippocampal LTD. In this study, we examined the effects of several ECs and other endogenous agents with actions at cannabinoid receptors, including anandamide, 2-arachidonylglycerol (2AG), and noladin ether (NLDE) to determine whether they mimic the effects of synthetic EC analogs on synaptic responses in the CA1 region of rat hippocampal slices. We also examined interactions among LFS-LTD, CHPG-LTD, and EC-LTD, and determined whether the various forms of LTD involve NMDAR, mGluR5, and/or CB1R activation.

\section{MATERIALS AND METHODS}

\section{Animals}

Protocols for animal use were approved by the Washington University Animal Studies Committee in accordance with the NIH guidelines for care and use of laboratory animals.

\section{Hippocampal Slice Preparation}

Hippocampal slices were prepared from postnatal day 30 to 32. Sprague-Dawley rats purchased from Harlen (Indianapolis, IN) (Zorumski et al, 1996). Rats were anesthetized with isoflurane and decapitated. Slices were cut transversely into $500-\mu \mathrm{m}$ slices using a rotary slicer in artificial cerebrospinal fluid (ACSF) containing (in $\mathrm{mM}$ ): $124 \mathrm{NaCl}$, $5 \mathrm{KCl}, 2 \mathrm{MgSO}_{4}, 2 \mathrm{CaCl}_{2}, 1.25 \mathrm{NaH}_{2} \mathrm{PO}_{4}, 22 \mathrm{NaHCO}_{3}$, and 10 glucose, bubbled with $95 \% \mathrm{O}_{2} / 5 \% \mathrm{CO}_{2}$ at $4-6{ }^{\circ} \mathrm{C}$. Acutely prepared slices were placed on mesh in $10-\mathrm{ml}$ beakers containing gassed ACSF and maintained for at least $1 \mathrm{~h}$ at $30{ }^{\circ} \mathrm{C}$ before experiments.

\section{Electrophysiology}

At the time of study, slices were transferred individually to a submerged recording chamber. Experiments were performed at $30^{\circ} \mathrm{C}$ with continuous bath perfusion of ACSF at $2 \mathrm{ml} / \mathrm{min}$. Extracellular recordings were obtained from the apical dendritic layer of the CA1 region for analysis of population EPSPs and from the cell body layer for analysis of population spikes (PSs). EPSPs were measured by their maximal slopes, and PSs were measured as a maximal height from the apex of the first positive peak to the most negative point of the spike. Using paired stimulations at an interval of $21 \mathrm{~ms}$, slices showing paired-pulse facilitation of EPSPs and paired-pulse depression of PS, indicators of good slice health, were selected for study (Tokuda et al, 2010). EPSPs were monitored by applying single stimuli to the Schaffer collateral pathway every $60 \mathrm{~s}$ at an intensity sufficient to elicit half-maximal responses. After establishing a stable baseline for at least $10 \mathrm{~min}$ and a control inputoutput curve, $1 \mathrm{~Hz} \times 900 \mathrm{~s}$ LFS using the same intensity stimulus was applied to induce electrical LTD (LFS-LTD). In other experiments, EC agonists and mGluR5 agonists were administered for $15 \mathrm{~min}$. Input-output curves were repeated $60 \mathrm{~min}$ after each conditioning. Signals were digitized and analyzed using PCLAMP software (Axon Instruments, Union City, CA).

\section{Chemicals}

DL-aminophosphonovaleric acid (APV), DHPG, CHPG, MPEP, WIN55,212-2 (WIN), arachidonyl-2'-chloroethylamide (ACEA), anandamide 2AG, and AM251 were purchased from Tocris Bioscience (Ellisville, MO). AM630 and URB-597 were purchased from Cayman Chemical (Ann Arbor, MI). NLDE was purchased from Tocris Bioscience, Cayman Chemical, and Enzo Life Sciences (Plymouth Meeting, PA). NLDE can be unstable and was only used when it was kept frozen until the time of an experiment. MPEP, WIN, ACEA, NLDE, URB-597, and 2AG were stored in ethanol. AM630 was dissolved in $\mathrm{HCl}$. APV and CHPG were dissolved in $\mathrm{NaOH}$. CHPG used in this study was purchased after 2007 because results with CHPG purchased before this date were inconsistent.

\section{Data Analysis}

Data are expressed as mean \pm SEM and are expressed as percentage of baseline control responses (set at 100\%). In these studies, ' $n$ ' represents the number of slices studied in a given condition, and, unless stated otherwise, data were normalized with respect to initial control responses. Points in the graphs without error bars have SEM smaller than the symbol size. Statistical comparisons are based on analysis of input-output curves at baseline and $60 \mathrm{~min}$ after LFS or drug exposure, and represent the degree of change at the half-maximal point on the input half output curves compared with baseline responses. In some experiments, we also analyzed paired-pulse plasticity using baseline stimulation intensity to determine whether presynaptic changes contribute to LTD. As EPSPs are depressed after LTD induction, we also analyzed paired-pulse ratios using a higher-intensity stimulus that produced EPSPs comparable 
to those measured during initial baseline recordings. Where appropriate, Student's $t$-test was used for comparisons between two groups. If a test of equal variance failed, the non-parametric Mann-Whitney rank-sum test was applied. Statistical analyses were performed using commercial software (SigmaStat 3.11; Systat Software, Richmond, CA). $P$-values $<0.05$ were considered statistically significant.

\section{RESULTS}

As reported by others (Xu et al, 2010), 15 min administration of $5 \mu \mathrm{M}$ WIN, a synthetic cannabinoid agonist that activates both CB1Rs and CB2Rs, induced a persistent depression of synaptic responses in the CA1 region (EPSP slope: $47.7 \pm 7.9 \%$ of control EPSPs $60 \mathrm{~min}$ after WIN washout, $N=4$, data not shown). WIN-induced LTD was not altered by co-administration of $30 \mu \mathrm{M} \mathrm{D,L-APV,} \mathrm{a}$ competitive NMDAR antagonist $(51.0 \pm 3.5 \%, \quad N=3$, Figure 1a), although the same concentration of APV administered during the delivery of 900 pulse $1 \mathrm{~Hz}$ LFS completely blocked homosynaptic LTD induction (98.5 $\pm 0.7 \%$ of control, $N=3$, data not shown). WINinduced LTD was blocked completely by $5 \mu \mathrm{M}$ AM251, a selective CB1R inhibitor (EPSPs $60 \mathrm{~min}$ after washout; $103.0 \pm 5.1 \%, N=5, P<0.001$, Figure $1 \mathrm{~b})$. Continuous administration of AM251 after washout of WIN failed to prevent the depression induced by WIN $(47.1 \pm 9.1 \%, N=3$, data not shown), indicating that WIN-LTD does not result from ongoing CB1R activation. At $5 \mu \mathrm{M}$, AM630, a CB2R antagonist, had no significant effect on WIN-LTD
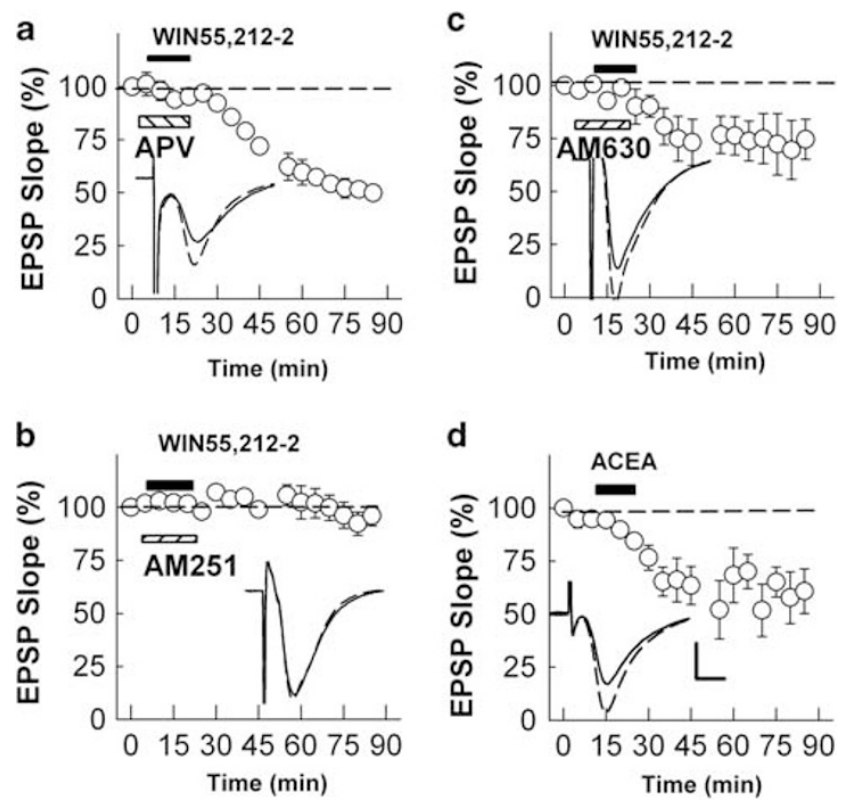

Figure I Chemical LTD induced by synthetic EC analogs. (a) Administration of $5 \mu \mathrm{M}$ WIN55,212-2 for $15 \mathrm{~min}$ (black bar) induced persistent depression (LTD) in the presence of $30 \mu \mathrm{M} \mathrm{APV.} \mathrm{(b,} \mathrm{c)} \mathrm{The}$ ability of WIN to induce LTD was blocked by co-administration of $5 \mu \mathrm{M}$ AM25I, a CBIR antagonist (panel b, hatched bar), but not by $5 \mu$ M AM630, a CB2R antagonist (panel $c$, hatched bar). (d). Administration of $5 \mu \mathrm{M}$ ACEA for 15 min (black bar) also induced persistent depression. Traces depict EPSPs before (dashed lines) and $60 \mathrm{~min}$ after administration of synthetic EC analogs. Scale; I $\mathrm{mV}$ and $5 \mathrm{~ms}$.
$(74.5 \pm 9.5 \%, N=6, P=0.305$, Figure 1c). These observations suggest that activation of CB1Rs, but not CB2Rs, drives a form of chemical LTD in the CA1 region. Consistent with this, 15 min administration of $5 \mu \mathrm{M}$ ACEA, another synthetic cannabinoid with relative selectivity for CB1Rs that induces LTD in corticostriatal synapses (Sergeeva et al, 2007), also induced persistent synaptic depression (Figure 1d).

These observations suggest that central nervous system (CNS)-generated ECs may also induce or contribute to persistent synaptic depression of glutamate transmission. This prompted us to examine several ECs and other endogenous cannabinoid receptor ligands for their ability to mimic the effects of WIN and ACEA. Administration of $5 \mu \mathrm{M}$ anandamide $(92.3 \pm 4.9 \%$ change, data, $N=5$, Figure $2 \mathrm{a})$ or $30 \mu \mathrm{M}$ oleamide $(94.5 \pm 7.7 \%$ change, data, $N=3$, not shown) for $15 \mathrm{~min}$ had no significant effect on EPSPs. Anandamide also failed to induce robust LTD in slices pretreated with $0.3 \mu \mathrm{M}$ URB-597, a fatty-acid amide hydrolase inhibitor $(85.8 \pm 8.1 \%, N=7, P=0.343$ vs anandamide alone by Mann-Whitney test). Although $5 \mu \mathrm{M} 2 \mathrm{AG}$ depressed EPSPs, the degree of persistent LTD was marginal $(90.1 \pm 12.7 \%, N=5$, Figure $2 \mathrm{~b})$. A higher concentration of 2AG $(20 \mu \mathrm{M})$ induced only slightly greater synaptic depression $(82.4 \pm 4.7 \%, N=3)$ that was less than the depression observed with WIN or ACEA. In contrast, administration of $5 \mu \mathrm{M}$ NLDE slowly but persistently depressed EPSPs $(69.7 \pm 5.2 \%, N=3$, Figure $2 \mathrm{c})$. Owing to the more robust and reliable effects of NLDE, we explored its actions in greater detail and found that a similar degree of LTD could be induced at concentrations as low as $0.1 \mu \mathrm{M}$. We thus used $0.1 \mu \mathrm{M}$ NLDE for all subsequent studies. LTD induced by NLDE seemed to be saturated by a single administration because a second administration of NLDE did not induce further LTD (EPSP slope: $54.0 \pm 7.3 \% 60 \mathrm{~min}$ after the first NLDE administration and $60.2 \pm 7.5 \% 60 \mathrm{~min}$ after the second NLDE administration, $N=5$ ).

These results indicate that certain cannabinoids, acting through CB1Rs induce a form of chemical LTD that does not involve NMDARs, and NLDE is an endogenous candidate to participate in LTD resulting from stimulation of the Schaffer collateral pathway. As Schaffer collateral LTD can also involve mGluR5, we examined whether cannabinoid-induced LTD shares mechanisms with mGluR-induced LTD. For these studies, we used CHPG, a selective mGluR5 agonist that has previously been shown to induce chemical LTD (Lanté et al, 2006). At $250 \mu \mathrm{M}, \mathrm{CHPG}$ acutely suppressed EPSPs and resulted in synaptic depression that persisted after washout $(75.6 \pm 3.6 \%, 60 \mathrm{~min}$ after CHPG, $N=11$, Figure $3 \mathrm{a}$ and c). Repeated administrations of CHPG did not result in further LTD $(71.5 \pm 6.4 \% 60 \mathrm{~min}$ after the first CHPG administration and $71.2 \pm 6.3 \% 60 \mathrm{~min}$ after the second CHPG administration, $N=6$ ). Administration of NLDE after induction of stable LTD with CHPG also failed to produce further LTD (Figure 3a). Similarly, administration of CHPG after establishing NLDE-LTD resulted in no further lasting change in synaptic responses (Figure $3 \mathrm{~b}$ ), suggesting that these two forms of LTD share common mechanisms.

Delivery of Schaffer collateral LFS after induction of CHPG-LTD also failed to produce further LTD (69.9 $\pm 5.8 \%$ after CHPG and $69.6 \pm 5.2 \%$ after LFS, $N=5$, Figure $3 \mathrm{c}$ ), 

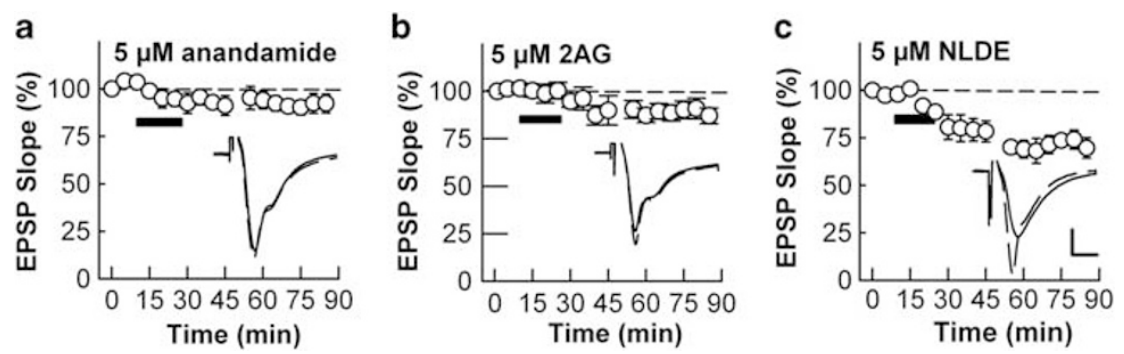

Figure 2 Effects of exogenously applied ECs on synaptic transmission. (a) Administration of $5 \mu$ M anandamide for 15 min (black bar) had marginal effects on EPSPs. (b) Administration of $5 \mu$ M 2AG for 15 min (hatched bar) only partially depressed EPSPs. (c) Administration of $5 \mu$ M NLDE for I 5 min (black bar) slowly depressed EPSPs with depression becoming even more prominent after washout. Traces depict EPSPs before (dashed lines) and 60 min after administration of each EC. Scale; I $\mathrm{mV}$ and $5 \mathrm{~ms}$.
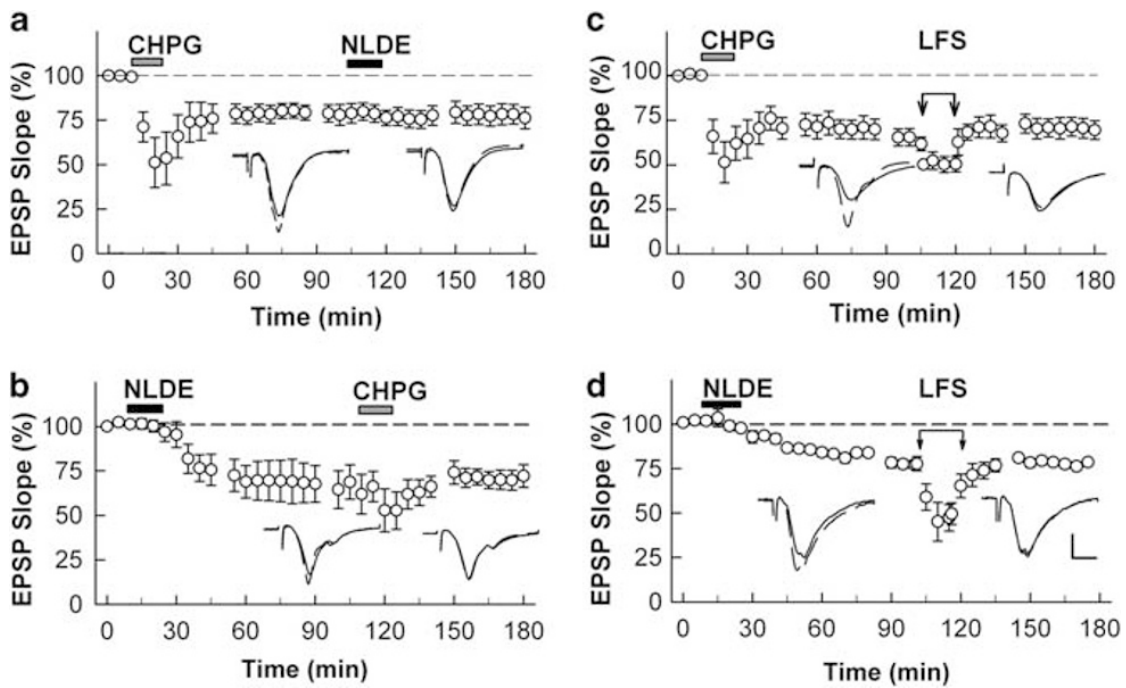

Figure 3 Non-additivity of LFS-induced LTD and two forms of chemically induced LTD. (a) Administration of $250 \mu M$ CHPG for I5 min (gray bar) acutely depressed EPSPs and resulted in sustained LTD. After induction of LTD with CHPG, 0.I $\mu M$ NLDE (black bar) failed to induce further LTD. (b) Administration of $0.1 \mu$ M NLDE for 15 min (black bar) slowly depressed EPSPs and resulted in LTD. After induction of NLDE-LTD, $250 \mu M$ CHPG (gray bar) failed to induce further LTD. (c) After induction of CHPG-LTD, LFS (I Hz for 15 min, arrows) failed to induce further LTD, although short-term depression was observed during LFS. (d) After induction of NLDE-LTD, LFS (arrows) failed to induce further LTD, although short-term depression was observed during delivery of LFS. Traces depict EPSPs before (dashed lines) and $60 \mathrm{~min}$ after each treatment. Scale; I mV and $5 \mathrm{~ms}$.

although LFS reliably induced LTD in naive slices $(70.5 \pm 9.0 \%, N=5$, see Figure 7). Moreover, LFS failed to induce further depression when administered after NLDELTD $(70.6 \pm 2.8 \%$ after NLDE and $71.3 \pm 2.4 \%$ after LFS, $N=5$, Figure $3 \mathrm{~d}$ ). Taken together, these data support the idea that these three forms of LTD share common induction mechanisms. We also examined whether these three forms of LTD have similar effects on paired-pulse plasticity. After stable LTD induction, all three forms of LTD resulted in a statistically significant increase in the paired-pulse ratio (Figure 4).

As LFS-LTD is known to require NMDAR activation (Dudek and Bear, 1992; Fujii et al, 1991), we examined whether other forms of LTD also involve NMDARs. Neither NLDE-LTD nor CHPG-LTD was altered by $30 \mu \mathrm{M}$ D,L-APV $(70.3 \pm 6.9 \%, 74.9 \pm 2.5 \%, N=5$, respectively. Figure $5 \mathrm{a}$ and b). Given the interactions among these forms of LTD and the fact that LFS-induced LTD is blocked completely by this concentration of APV, this suggests that activation of mGluR5 and CB1Rs likely occurs downstream of NMDARs in the cascade leading to LFS-LTD. To determine the role of
mGluR5 in these forms of LTD, we examined MPEP, a relatively selective mGluR5 antagonist. In the presence of $10 \mu \mathrm{M}$ MPEP, LFS failed to induce LTD $(97.8 \pm 3.1 \%, N=5$, Figure 6a). MPEP also blocked CHPG-induced LTD $(96.6 \pm 3.3 \%, N=5$, Figure $6 \mathrm{~b})$. In contrast, NLDE administered in the presence of MPEP resulted in a typical slowly developing form of LTD $(71.1 \pm 9.8 \%, N=5$, Figure $6 c)$. These results suggest that CB1R activation occurs downstream of mGluR5 in the LTD pathway.

To determine whether ECs act downstream of mGluR5, we examined the effects of AM251 against LFS, CHPG, and NLDE. All three forms of LTD were blocked by AM251 $(90.0 \pm 5.0 \%, 105.0 \pm 4.7 \%, 94.8 \pm 1.2 \%$, respectively, $N=5$ each, Figure $7 \mathrm{a}-\mathrm{c}$ ). In contrast, $5 \mu \mathrm{M}$ AM630, a specific CB2R antagonist, failed to block LTD induced by any of these treatments $(66.2 \pm 11.4 \%, 64.4 \pm 1.6 \%, 65.5 \pm 10.1 \%$, respectively, $N=3$ each, data not shown). Continuous administration of AM251 after washout of NLDE also failed to prevent NLDE-LTD $(77.1 \pm 4.1 \%, N=5$, Figure $7 \mathrm{~d})$, again indicating that the slowly developing LTD does not result from ongoing CB1R activation. 

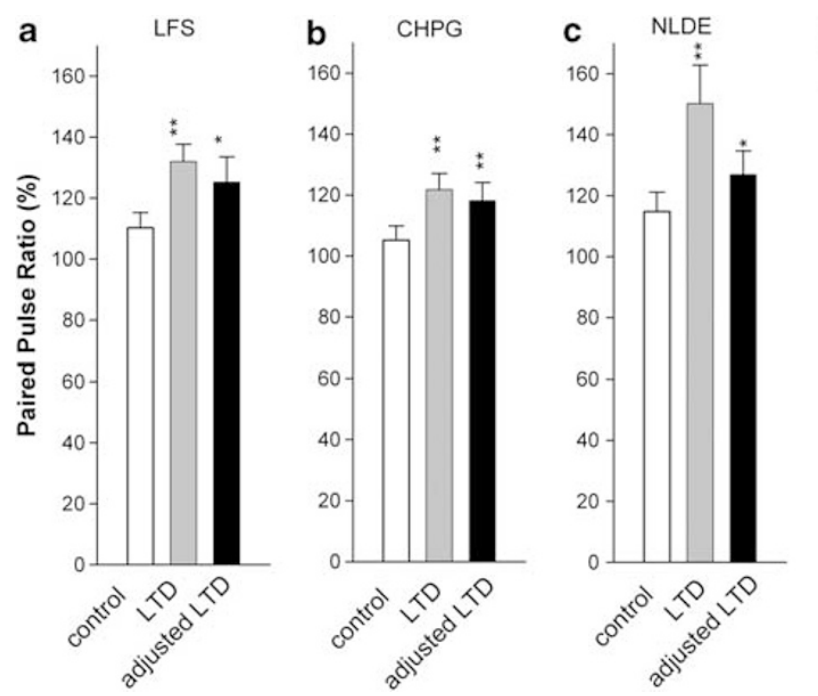

d

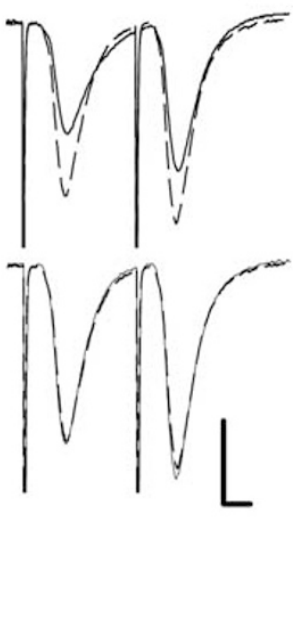

Figure 4 Effects of LTD on paired-pulse plasticity. The bar graphs show the effects of LFS $(a, N=\mid \mathrm{I}), \mathrm{CHPG}(\mathrm{b}, N=10)$, and NLDE (c, N=I0) on paired-pulse plasticity using an interpulse interval of $21 \mathrm{~ms}$. Under baseline conditions, a $50 \%$ maximal stimulus resulted in a small degree of paired-pulse facilitation (PPF) (white bars). Sixty minutes after LTD induction, all three treatments resulted in significantly increased PPF using this same stimulation (gray bars). Black bars show enhanced PPF after LTD even when the stimulus intensity was increased to activate EPSPs of the same size as in the initial baseline recordings. The traces to the right of the graphs (d) show raw EPSPs under baseline conditions (dashed traces) and after LFS induced LTD (solid traces). Upper traces use the baseline 50\% maximal stimulus, whereas bottom traces show PPF after increasing the stimulus intensity to generate a conditioning EPSP similar to that observed in the initial baseline recordings (solid traces). Scale bar: I mV, $5 \mathrm{~ms}$. ${ }^{*} P<0.05$, $* * P<0.0$ I, compared with baseline PPF.
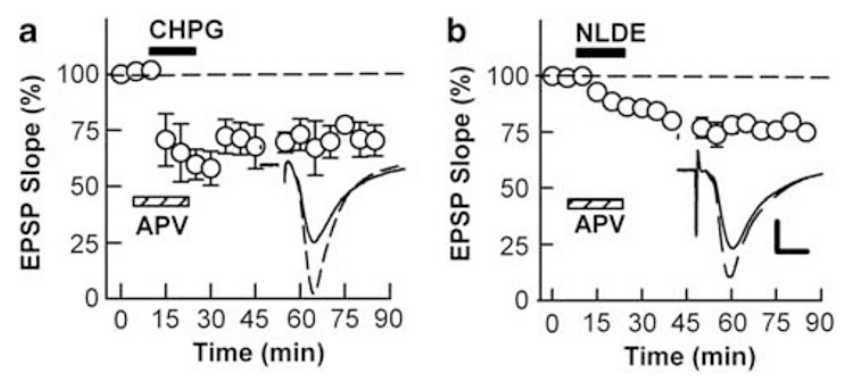

Figure 5 CHPG-LTD and NLDE-LTD do not require NMDAR activation. (a) Administration of $250 \mu$ M CHPG (black bar) in the presence of $30 \mu \mathrm{M}$ APV (hatched bar) successfully induced LTD. (b) Similarly, administration of $0.1 \mu M$ NLDE (black bar) in the presence of APV induced LTD. Traces depict EPSPs before (dashed lines) and $60 \mathrm{~min}$ after administration of CHPG and NLDE. Scale; I mV and $5 \mathrm{~ms}$.

As DHPG, a less selective group I mGluR agonist, has been reported to induce a unique form of LTD (Palmer $e t a l$, 1997), we also examined the sensitivity of DHPG-induced LTD to MPEP and AM251. In contrast to CHPG, we found that DHPG induced robust LTD in the presence of MPEP $(72.7 \pm 5.9 \%, N=5$, Figure $8 \mathrm{a})$, suggesting that this form of LTD is not mediated solely by activation of mGluR5. DHPGLTD was also induced in the presence of AM251 $(59.0 \pm 7.8 \%, N=6$, Figure $8 \mathrm{~b})$. LTD in the presence of MPEP and AM251 did not differ from DHPG alone $(62.6 \pm 8.7 \%, N=5)$. To examine interactions of DHPGLTD with other forms of LTD, CHPG and DHPG were administered sequentially after stable induction of LFSLTD. Although administration of CHPG did not induce further LTD after LFS-LTD, administration of DHPG induced additional and persistent synaptic depression (EPSP change $70.5 \pm 9.0 \% 60 \mathrm{~min}$ after LFS, $84.8 \pm 4.6 \%$
$60 \mathrm{~min}$ after CHPG administration, and $27.7 \pm 4.1 \% 60 \mathrm{~min}$ after DHPG administration, $N=5$, Figure 8c). These observations again suggest that LFS-LTD and CHPG-LTD share common mechanisms, but differ from DHPG-induced LTD. Moreover, DHPG induced further depression after establishing stable NDLE-LTD $(57.1 \pm 10.4 \%$ by NLDE and $32.8 \pm 7.2 \%$ by DHPG after NLDE, $P<0.05, N=4$, data not shown).

\section{DISCUSSION}

These results indicate that glutamate acting on NMDARs and mGluR5, and ECs acting on CB1Rs participate in the events underlying LTD induced by electrical stimulation of the Schaffer collateral pathway. On the basis of occlusion experiments and the effects of receptor antagonists, these studies further suggest that activation of glutamate receptors occurs upstream of cannabinoid receptor activation.

\section{Differences between CHPG- and DHPG-Induced LTD}

The ability of mGluR antagonists to inhibit LFS-induced LTD suggests that these receptors have a key role in the induction and/or maintenance of this form of synaptic plasticity. Furthermore, chemical LTD induced by DHPG, an agonist of group I mGluRs that includes mGluR1 and mGluR5 (Brabet et al, 1995), has been extensively studied, in part because of its ease of induction and its presumed likelihood to involve mechanisms similar to those evoked by synaptic stimulation. However, other investigators have suggested that LFS-induced LTD and DHPG-LTD may not involve the same mechanisms, and DHPG has been found to induce additional synaptic depression after induction of stable LFS-LTD (Palmer et al, 1997; Fitzjohn et al, 1999). 

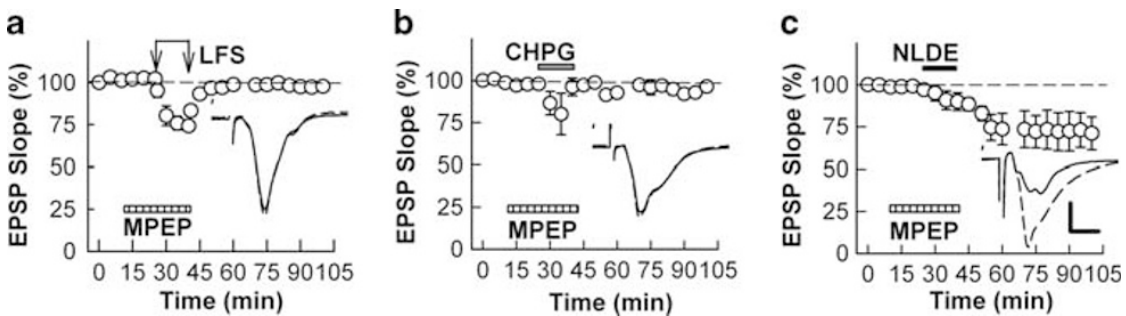

Figure 6 An mGluR5 antagonist blocks LFS-LTD and CHPG-LTD but not NLDE-LTD. (a) LFS (arrows) in the presence of I0 $\mu M$ MPEP (hatched bar) acutely depressed EPSPs but did not induce LTD. (b) Administration of $250 \mu$ M CHPG (gray bar) in the presence of MPEP failed to induce LTD. (c) In contrast, administration of $0.1 \mu \mathrm{M}$ NLDE (black bar) in the presence of MPEP resulted in a slowly developing form of LTD. Traces depict EPSPs before (dashed lines) and $60 \mathrm{~min}$ after administration of LFS, CHPG, and NLDE. Scale; I mV and $5 \mathrm{~ms}$.
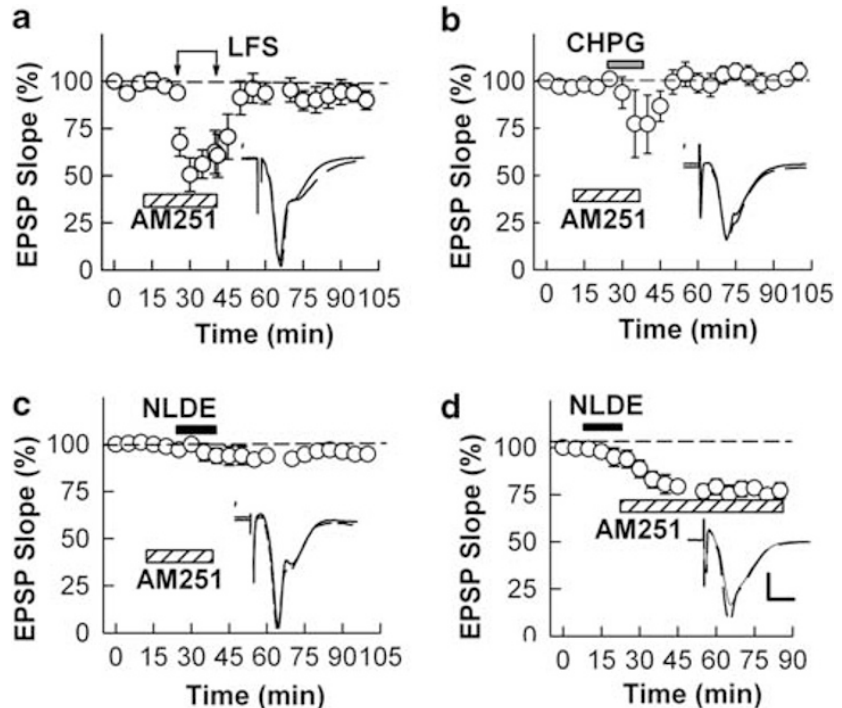

Figure 7 Effects of a CBIR antagonist on LTD. (a) LFS (arrows) in the presence of $5 \mu \mathrm{M}$ AM25I (hatched bar) acutely depressed EPSPs but did not induce LTD. (b) Administration of $250 \mu \mathrm{M}$ CHPG (gray bar) in the presence of AM25 I acutely depressed EPSPs but also failed to induce LTD. (c) AM25 I also blocked the ability of $0.1 \mu M$ NLDE (black bar) to induce LTD. (d) AM25 I administered after washout of NLDE did not prevent the depression. Traces depict EPSPs before (dashed lines) and $60 \mathrm{~min}$ after administration of LFS, CHPG, and NLDE. Scale; I $\mathrm{mV}$ and $5 \mathrm{~ms}$.

Furthermore, it has been recently reported that LFS-LTD can be induced after DHPG-LTD (Wang et al, 2008). CHPG, a more specific mGluR5 agonist (Doherty et al, 1997), has been used less often for LTD studies, but has been found to induce or augment LTD in several studies (Palmer et al, 1997; Lanté et al, 2006; Neyman and Manahan-Vaughan, 2008). In this study, we found that LFS and CHPG occlude each other's effects but that DHPG induces additional synaptic depression after induction of LFS-LTD or CHPG-LTD.

These results strongly suggest that DHPG-LTD differs mechanistically from CHPG-LTD, despite the fact that DHPG is an agonist at group I mGluRs. Consistent with this, MPEP, an mGluR5 antagonist, completely inhibits CHPG-LTD and LFS-LTD but not DHPG-LTD, again suggesting that these forms of LTD involve different mechanisms. A previous study found that DHPG-induced LTD is blocked by the group II mGluR antagonist, LY341495, suggesting that mGluR2 and/or mGluR3 has an
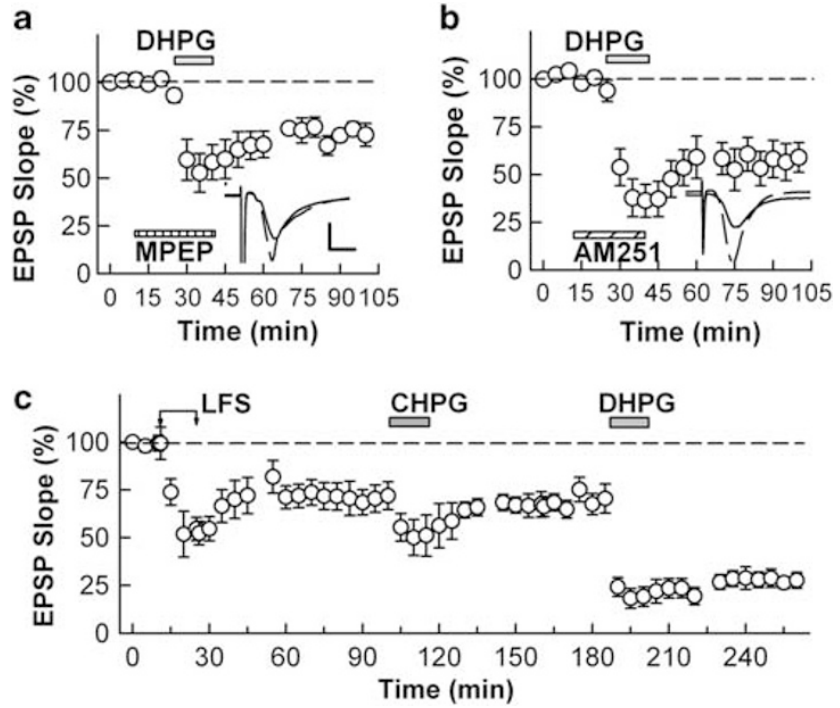

Figure 8 DHPG-LTD is insensitive to mGluR5 and CBIR antagonists and shows additivity with LFS-induced LTD. (a) Administration of $100 \mu \mathrm{M}$ DHPG (gray bar) with $10 \mu$ M MPEP (hatched bar) acutely depressed EPSPs and the depression persisted after washout. (b) Similarly, administration of DHPG in the presence of AM25 I induced LTD. (c) In naive slices, LFS (arrows) readily induced LTD. After LFS-induced LTD, administration of $250 \mu \mathrm{M}$ CHPG (left gray bar) acutely depressed EPSPs but failed to induce further LTD. In contrast, administration of $100 \mu \mathrm{M}$ DHPG (right gray bar) resulted in additional LTD. Traces depict EPSPs before (dashed lines) and $60 \mathrm{~min}$ after each treatment. Scale; $1 \mathrm{mV}$ and $5 \mathrm{~ms}$.

important role (Watabe et al, 2002). Moreover, others have found that DHPG does not involve ECs (Rouach and Nicoll, 2003) and may induce a form of synaptic loss rather than traditional synaptic plasticity, particularly after repeated applications (Kamikubo et al, 2006; Shinoda et al, 2010). At the minimum, we conclude that DHPG-LTD does not simply result from activation of mGluR5 and thus should be considered separately from other forms of LTD. In contrast, CHPG-induced LTD seems to share key mechanisms with LFS-LTD, and highlights the importance of mGluR5 activation in homosynaptic plasticity.

\section{Involvement of ECs in Hippocampal LTD}

Consistent with the above discussion, previous studies have found that SR141716 (Rimonabant), a CB1R antagonist, does not block DHPG-induced LTD in the CA1 region 
(Rouach and Nicoll, 2003), but does attenuate LFS-LTD (Xu et al, 2010). This suggests a key role for CB1Rs in conventional LTD resulting from Schaffer collateral pathway stimulation. Although it is widely accepted that ECs regulate GABAergic neurotransmission through CB1Rs, the role of ECs in glutamatergic synaptic plasticity is less certain (Misner and Sullivan, 1999). Early studies indicated that CB1Rs are not expressed on glutamatergic neurons (Katona et al, 1999; Hájos et al, 2000; Hoffman and Lupica, 2000), and that the synthetic cannabinoid agonist, WIN, either did not depress excitatory synaptic responses (AlHayani and Davies, 2000) or, in studies in which it did, the CB1R antagonist, AM251, failed to prevent its effects (Hajos and Freund, 2002). Recent studies have found that WIN does depress hippocampal EPSPs and provide support for the idea that excitatory synaptic transmission is directly modulated by CB1Rs on glutamatergic neurons (Domenici et al, 2006; Xu et al, 2010). Our findings are consistent with these latter studies. In our study, WIN-induced depression persisted after drug washout, resembling LFS-induced LTD. WIN-LTD was blocked by co-administration of AM251 but not by continuous administration of AM251 during drug washout, suggesting that CB1Rs are involved in the induction of WIN-LTD but that the depression does not require ongoing receptor stimulation. Furthermore, our results indicate that AM251 inhibits LFS-LTD, indicating that activation of CB1Rs is involved in the induction of homosynaptic LTD at Schaffer collateral synapses.

Although early studies reported that CB2Rs are not found in the CNS, recent evidence indicates their expression in multiple brain regions including the hippocampus (Brusco et al, 2008a, b). Thus, CB2Rs are also a possible target for ECs in regulating synaptic function. In our study, the CB2R antagonist, AM630, did not block LTD induced by LFS, CHPG, WIN, or NLDE, suggesting that CB2Rs are unlikely to have a major role in any of these forms of LTD.

The ability of CB1R antagonists to block LFS-LTD raises important questions about which ECs mediate these effects. Multiple ECs and other ligands acting at cannabinoid receptors are synthesized in the CNS and these various messengers may have unique roles in modulating synaptic function (Banni and Di Marzo, 2010). NLDE is an EC that specifically activates CB1Rs, whereas anandamide has greater selectivity for CB2Rs and may have partial agonist effects at both receptor types. 2AG is believed to activate both CBRs at similar concentrations. Our results indicate that CB1R activation is necessary for LFS-induced LTD in the CA1 region. However, the pathway leading to LTD may be complex because $2 \mathrm{AG}$, a major $\mathrm{EC}$ in the CNS that acts at CB1Rs, did not induce substantial synaptic depression at concentrations up to $20 \mu \mathrm{M}$. It is possible that effects on CB2Rs are involved in limiting the actions of 2AG, although there may be other mechanisms to explain its diminished efficacy, including factors that regulate the production and metabolism of ECs. In our experiments, we found that submicromolar concentrations of NLDE induced a slowly developing form of LTD without producing significant depression during acute administration. The lack of acute effects of NLDE makes it difficult to determine its potential role in LTD. Furthermore, NLDE is a relatively unstable chemical, making studies of its effects even more complicated. Despite these problems, we found that freshly prepared solutions of NLDE slowly and persistently depress EPSPs by acting at CB1Rs and it must be noted that NLDE is found endogenously in the hippocampus (Fezza et al, 2002).

The acute depression observed during administration of CHPG or during delivery of LFS is likely mediated by mechanisms that differ from those underlying longerlasting synaptic depression. A previous study found that acute depression induced by $10 \mu \mathrm{M}$ DHPG is blocked by MPEP (Piccinin et al, 2010), suggesting a role for mGluR5, although the long-lasting depression after DHPG administration seems to involve more complex mechanisms as outlined above (Fitzjohn et al, 1999). In our study, acute depression induced by CHPG was largely but not completely eliminated by MPEP, suggesting that although mGluR5 is involved in acute depression, this receptor may not be the only participant. In contrast, acute depression during delivery of LFS was not attenuated by MPEP, suggesting that other mechanisms account for acute depression during synaptic stimulation.

\section{LTD Induction Mechanisms}

LTD induced by LFS, CHPG, and NLDE seems to share common mechanisms, based on the finding that these forms of LTD are non-additive. However, only LFS-LTD is blocked by APV, suggesting that activation of NMDARs occurs at very early stages of the LTD induction process. Furthermore, NMDARs are directly activated by LFS (Dudek and Bear, 1992; Fujii et al, 1991) but not by activation of mGluR5 or CB1Rs. Consistent with this, CHPG-LTD and NLDE-LTD are not altered by APV. In contrast, MPEP inhibits both LFS-LTD and CHPG-LTD, but not NLDE-LTD or WIN-LTD. Our observations suggest that NMDARs and mGluR5 act early in the sequence leading to LFS-LTD. The lack of effect of APV and MPEP on cannabinoid-induced LTD suggests that release of ECs occurs later in the pathway and is consistent with data indicating that WIN depresses EPSPs in the presence of APV (Domenici et al, 2006).

Taken together, our results indicate that three receptormediated components involved in LFS-induced LTD in the CA1 region, namely NMDAR activation, mGluR5 activation, and EC-driven CB1R stimulation, do not function independently, but rather work in concert with each other in a cascade that promotes lasting synaptic change. Among these, it seems that NMDAR and mGluR5 activation occur early in the process followed by release of ECs (Figure 9). Yasuda et al (2008) proposed a similar scheme to explain heterosynaptic LTD in the visual cortex. In their scenario, postsynaptic activation of mGluR5 stimulates the subsequent release of ECs as retrograde messengers that activate presynaptic CB1Rs and depress neurotransmitter release. This scheme also seems to be relevant to LTD in the CA1 region of the hippocampus (Chevaleyre and Castillo, 2003). In our model, we suggest that ECs act retrogradely to alter presynaptic function, consistent with previous reports (Heifets and Castillo, 2009; Yasuda et al, 2008). In all three forms of LTD we studied, we observed changes in pairedpulse plasticity that are consistent with a presynaptic contribution to LTD. However, we note that ECs also have postsynaptic actions and effects on non-pyramidal cells (Heifets and Castillo, 2009), and that LFS-induced LTD likely involves both postsynaptic and presynaptic changes 


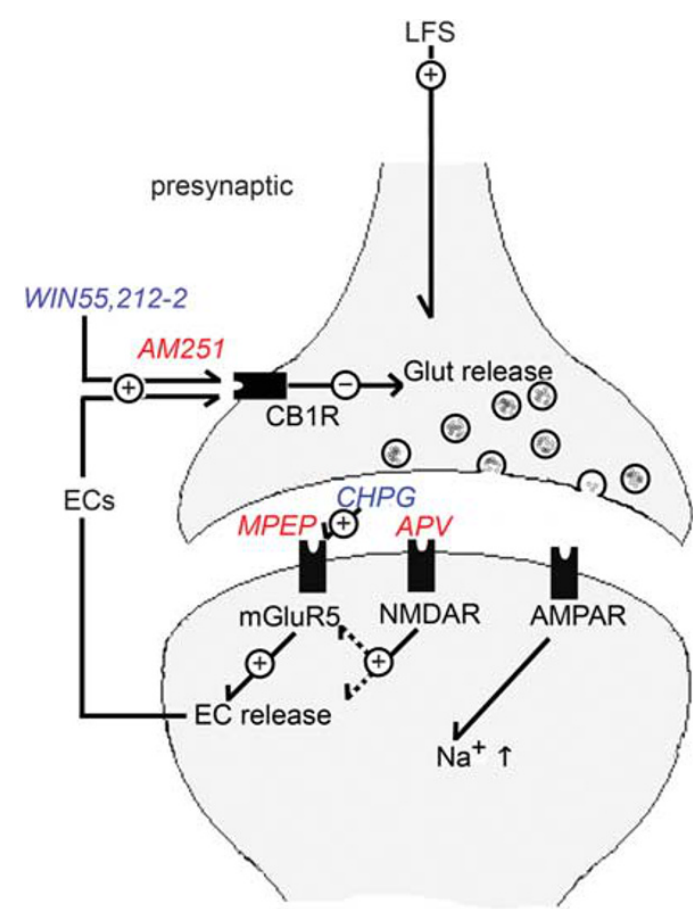

Figure 9 LTD induced by LFS, CHPG, and ECs share common mechanisms. APV blocks only LFS-induced LTD, whereas MPEP blocks both LFS-LTD and CHPG-LTD, but not NLDE-LTD. AM25 I blocks all three forms of LTD. These results suggest that NMDARs, mGluR5, and CBIRs participate in a cascade that drives LTD induction. Present data suggest that synaptic stimulation activates NMDARs and mGluR5 early in the process and that release of ECs and CBIR activation occur later.

in function (Luscher and Huber, 2010; Poschel and Stanton, 2007). Although our study has focused on homosynaptic LTD induced by single-pulse $1 \mathrm{~Hz}$ stimulation, future studies examining heterosynaptic depression in the CA1 region are required to determine the roles that ECs have in other forms of synaptic modulation. Owing to the role that LTD is believed to have in memory and the roles that ECs may have in the pathophysiology of multiple psychiatric illnesses, including substance abuse syndromes, mood and anxiety disorders, and psychosis (D'Souza, 2007; Leweke and Koethe, 2008; Witkin et al, 2005), our results have implications for understanding the cognitive changes that accompany neuropsychiatric disorders.

\section{ACKNOWLEDGEMENTS}

This study was supported in part by NIH grants MH07791 and AA017413 and the Bantly Foundation.

\section{DISCLOSURE}

Dr Zorumski serves as a consultant for Sage Therapeutics. Otherwise, Drs Izumi and Zorumski have no potential conflicts of interest, nor any financial interests to disclose.

\section{REFERENCES}

Abush H, Akirav I (2010). Cannabinoids modulate hippocampal memory and plasticity. Hippocampus 20: 1126-1138.
Al-Hayani A, Davies SN (2000). Cannabinoid receptor mediated inhibition of excitatory synaptic transmission in the rat hippocampal slice is developmentally regulated. Br J Pharmacol 131: 663-665.

Banni S, Di Marzo V (2010). Effect of dietary fat on endocannabinoids and related mediators: consequences on energy homeostasis, inflammation and mood. Mol Nutr Food Res 54: 82-92.

Bashir ZI, Jane DE, Sunter DC, Watkins JC, Collingridge GL (1993). Metabotropic glutamate receptors contribute to the induction of long-term depression in the CA1 region of the hippocampus. Eur J Pharmacol 239: 265-266.

Bellone C, Lüscher C, Mameli M (2008). Mechanisms of synaptic depression triggered by metabotropic glutamate receptors. Cell Mol Life Sci 65: 2913-2923.

Bolshakov VY, Siegelbaum SA (1994). Postsynaptic induction and presynaptic expression of hippocampal long-term depression. Science 264: 1148-1152.

Brabet I, Mary S, Bockaert J, Pin JP (1995). Phenylglycine derivatives discriminate between mGluR1- and mGluR5mediated responses. Neuropharmacology 34: 895-903.

Brusco A, Tagliaferro PA, Saez T, Onaivi ES (2008a). Ultrastructural localization of neuronal brain CB2 cannabinoid receptors. Ann NY Acad Sci 1139: 450-457.

Brusco A, Tagliaferro P, Saez T, Onaivi ES (2008b). Postsynaptic localization of CB2 cannabinoid receptors in the rat hippocampus. Synapse 62: 944-949.

Chevaleyre V, Castillo PE (2003). Heterosynaptic LTD of hippocampal GABAergic synapses: a novel role of endocannabinoids in regulating excitability. Neuron 38: 461-472.

Doherty AJ, Palmer MJ, Henley JM, Collingridge GL, Jane DE (1997). (RS)-2-chloro-5-hydroxyphenylglycine (CHPG) activates mGlu5, but not mGlu1, receptors expressed in CHO cells and potentiates NMDA responses in the hippocampus. Neuropharmacology 36: 265-267.

Domenici MR, Azad SC, Marsicano G, Schierloh A, Wotjak CT, Dodt HU et al (2006). Cannabinoid receptor type 1 located on presynaptic terminals of principal neurons in the forebrain controls glutamatergic synaptic transmission. J Neurosci 26: 5794-5799.

D’Souza DC (2007). Cannabinoids and psychosis. Int Rev Neurobiol 78: 289-326.

Dudek SM, Bear MF (1992). Homosynaptic long-term depression in area CA1 of hippocampus and effects of N-methyl-D-aspartate receptor blockade. Proc Natl Acad Sci USA 89: 4363-4367.

Fezza F, Bisogno T, Minassi A, Appendino G, Mechoulam R, Di Marzo V (2002). Noladin ether, a putative novel endocannibinoid: inactivation mechanisms and a sensitive method for its quantification in rat tissues. FEBS Lett 513: 294-298.

Fitzjohn SM, Kingston AE, Lodge D, Collingridge GL (1999). DHPG-induced LTD in area CA1 of juvenile rat hippocampus; characterisation and sensitivity to novel mGlu receptor antagonists. Neuropharmacology 38: 1577-1583.

Fujii S, Saito K, Miyakawa H, Ito K, Kato H (1991). Reversal of long-term potentiation (depotentiation) induced by tetanus stimulation of the input to CA1 neurons of guinea pig hippocampal slices. Brain Res 555: 112-122.

Gerdeman GL, Ronesi J, Lovinger DM (2002). Postsynaptic endocannabinoid release is critical to long-term depression in the striatum. Nat Neurosci 5: 446-451.

Gladding CM, Fitzjohn SM, Molnár E (2009). Metabotropic glutamate receptor-mediated long-term depression: molecular mechanisms. Pharmacol Rev 61: 395-412.

Hajos N, Freund TF (2002). Pharmacological separation of cannabinoid sensitive receptors on hippocampal excitatory and inhibitor fibers. Neuropharmacology 43: 503-510.

Hájos N, Katona I, Naiem SS, MacKie K, Ledent C, Mody I et al (2000). Cannabinoids inhibit hippocampal GABAergic transmission and network oscillations. Eur J Neurosci 12: 3239-3249. 
Heifets BD, Castillo PE (2009). Endocannabinoid signaling and long-term synaptic plasticity. Annu Rev Neurosci 71: 283-306.

Hoffman AF, Lupica CR (2000). Mechanisms of cannabinoid inhibition of GABA(A) synaptic transmission in the hippocampus. J Neurosci 20: 2470-2479.

Huang Y, Yasuda H, Sarihi A, Tsumoto T (2008). Roles of endocannabinoids in heterosynaptic long-term depression of excitatory synaptic transmission in visual cortex of young mice. J Neurosci 28: 7074-7083.

Huber KM, Roder JC, Bear MF (2001). Chemical induction of mGluR5- and protein synthesis - dependent long-term depression in hippocampal area CA1. J Neurophysiol 86: 321-325.

Kamikubo Y, Egashira Y, Tanaka T, Shinoda Y, Tominaga-Yoshino K, Ogura A (2006). Long-lasting synaptic loss after repeated induction of LTD: independence to the means of LTD induction. Eur J Neurosci 24: 1606-1616.

Katona I, Sperlágh B, Sík A, Käfalvi A, Vizi ES, Mackie K et al (1999). Presynaptically located CB1 cannabinoid receptors regulate GABA release from axon terminals of specific hippocampal interneurons. J Neurosci 19: 4544-4558.

Kemp A, Manahan-Vaughan D (2004). Hippocampal long-term depression and long-term potentiation encode different aspects of novelty acquisition. Proc Natl Acad Sci USA 101: 8192-8197.

Lanté F, Cavalier M, Cohen-Solal C, Guiramand J, Vignes M (2006). Developmental switch from LTD to LTP in low frequencyinduced plasticity. Hippocampus 16: 981-989.

Leweke FM, Koethe D (2008). Cannabis and psychiatric disorders: it is not only addiction. Addict Biol 13: 264-275.

Luscher C, Huber KM (2010). Group 1 mGluR-dependent synaptic long-term depression: mechanisms and implications for circuitry and disease. Neuron 65: 445-459.

Malenka RC, Bear MF (2004). LTP and LTD: an embarrassment of riches. Neuron 44: 5-21.

Massey PV, Bashir ZI (2007). Long-term depression: multiple forms and implications for brain function. Trends Neurosci 30: $176-184$.

Misner DL, Sullivan JM (1999). Mechanism of cannabinoid effects on long-term potentiation and depression in hippocampal CA1 neurons. J Neurosci 19: 6795-6805.

Neyman S, Manahan-Vaughan D (2008). Metabotropic glutamate receptor 1 (mGluR1) and 5 (mGluR5) regulate late phases of LTP and LTD in the hippocampal CA1 region in vitro. Eur J Neurosci 27: $1345-1352$.

Overstreet LS, Pasternak JF, Colley PA, Slater NT, Trommer BL (1997). Metabotropic glutamate receptor mediated long-term depression in developing hippocampus. Neuropharmacology 36: 831-844.

Palmer MJ, Irving AJ, Seabrook GR, Jane DE, Collingridge GL (1997). The group I mGlu receptor agonist DHPG induces a novel form of LTD in the CA1 region of the hippocampus. Neuropharmacology 36: 1517-1532.

Piccinin S, Cinque C, Calo L, Molinaro G, Battaglia G, Maggi L et al (2010). Interaction between ephrins and mGluR5 metabotropic glutamate receptors in the induction of long-term potentiation in the hippocampus. J Neurosci 30: 2835-2843.

Poschel B, Stanton PK (2007). Comparison of cellular mechanisms of long-term depression of synaptic strength at perforant pathgranule cell and Schaffer collateral-CA1 synapses. Prog Brain Res 163: $473-500$

Reyes-Harde M, Stanton PK (1998). Postsynaptic phospholipase C activity is required for the induction of homosynaptic long-term depression in rat hippocampus. Neurosci Lett 252: 155-158.

Rouach N, Nicoll RA (2003). Endocannabinoids contribute to short-term but not long-term mGluR-induced depression in the hippocampus. Eur J Neurosci 18: 1017-1020.

Selig DK, Hjelmstad GO, Herron C, Nicoll RA, Malenka RC (1995). Independent mechanisms for long-term depression of AMPA and NMDA responses. Neuron 15: 417-426.

Sergeeva OA, Doreulee N, Chepkova AN, Kazmierczak T, Haas HL (2007). Long-term depression of cortico-striatal synaptic transmission by DHPG depends on endocannabinoid release and nitric oxide synthesis. Eur J Neurosci 26: 1889-1894.

Shinoda Y, Tanaka T, Tominaga-Yoshino K, Ogura A (2010). Persistent synapse loss induced by repetitive LTD in developing rat hippocampal neurons. PLoS One 5: e10390.

Stanton PK, Chattarji S, Sejnowski TJ (1991). 2-Amino-3phosphonopropionic acid, an inhibitor of glutamate-stimulated phosphoinositide turnover, blocks induction of homosynaptic long-term depression, but not potentiation, in rat hippocampus. Neurosci Lett 127: 61-66.

Staubli U, Lynch G (1990). Stable depression of potentiated synaptic responses in the hippocampus with $1-5 \mathrm{~Hz}$ stimulation. Brain Res 513: 113-118.

Tokuda K, O’Dell, KA, Izumi Y, Zorumski CF (2010). Midazolam inhibits hippocampal long-term potentiation and learning through dual central and peripheral benzodiazepine receptor activation and neurosteroidogenesis. I Neuroscience 30: $16788-16795$.

Wang W, Zhang Z, Shang J, Jiang ZZ, Wang S, Liu Y et al (2008). Activation of group I metabotropic glutamate receptors induces long-term depression in the hippocampal CA1 region of adult rats in vitro. Neurosci Res 62: 43-50.

Watabe AM, Carlisle HJ, O'Dell TJ (2002). Postsynaptic induction and presynaptic expression of group $1 \mathrm{mGluR}$-dependent LTD in the hippocampal CA1 region. J Neurophysiol 87: 1395-1403.

Witkin JM, Tzavara ET, Nomikos GG (2005). A role for cannabinoid CB1 receptors in mood and anxiety disorders. Behav Pharmacol 16: 315-331.

Xu JY, Chen R, Zhang J, Chen C (2010). Endocannabinoids differentially modulate synaptic plasticity in rat hippocampal CA1 pyramidal neurons. PLoS One 5: e10306.

Yasuda H, Huang Y, Tsumoto T (2008). Regulation of excitability and plasticity by endocannabinoids and PKA in developing hippocampus. Proc Natl Acad Sci USA 105: 3106-3111.

Zorumski CF, Mennerick S, Izumi Y (1996). Assessment of synaptic effects of nitric oxide in hippocampal neurons. Methods Neurosci 31: 282-299. 